\title{
X-Ray Irradiator
}

National Cancer Institute

\section{Source}

National Cancer Institute. X-Ray Irradiator. NCI Thesaurus. Code C158341.

A device that exposes samples to X-ray radiation. 